\title{
A proof of the Legendre and Goldbach conjectures via a generalization of the concept of prime number
}

\author{
Jamel Ghanouchi
}

Professor of Mathematics

RIME Department of Mathematics

6 Rue Khansa 2070 Marsa Tunisia

Keywords : Primes ; Legendre ; Goldbach ; Conjectures ; Proof.

\section{Abstract}

In this paper, we generalize the concept of prime number and define new the new concept to the Legendre and Goldbach conjectures and to prove

\section{Introduction}

The prime numbers are called primes because they are the Micn of the n. $\mathrm{n}$ : Each number $n$ can be written $\prod_{j} p_{j}{ }^{n_{j}}$ when where $\mathrm{j} \mathrm{p}$ are primes and are integers. This writing is called the decomposition in prime factors of the number $n$.

In fact, this definition is a very particular case of a much

Indeed, if $n_{j}$ are rationals, everything changes.

Considering that the decomposition in prime fact of at integer $\mathrm{n}$ when $n_{j}$ are rationals $\prod_{j} p_{j}^{n_{j}}$. In this writhing, then the ${ }_{j}$ have no rea

For example, if we decide that 6 be written according to $16 \mathrm{a}$ d its ratio exponent instead of 2 .

If we decide conventiona 1 y number is prime, and it is possible by the fact that they are coprime two by tw then new prime (new primes=bricks with rational exponents in the writing) replaces an mer one in th of the old primes (old primes=bricks with integral exponents in the writing).

Example : If by co ntion, $t$ fifth Fermat number $F_{5}=2^{2^{5}}+1=4294967297=641.6700417$ is prime, w ecide it eplaces 641 which becomes compound when 6700417 remains prime or $641 \mathrm{re}$ ains $\mathrm{p}$ me and replaces 67004147 which becomes compound.

In all $\mathrm{c}_{\mathrm{a}}$ age is that we have a formula which gives for each $\mathrm{n}$ a prime. And we can see the the pri are infinite. There is anot Interesting result : Let Ulam spiral. The Fermat numbers are all situated in the same
line.

\section{The Legendre conjecture}

The Legendre conjecture states that there is always a prime number between the squares of two consecutive integers. So $\exists \mathrm{p} \mid \mathrm{n}^{2} \leq p \leq(n+1)^{2} ; \forall n \in N$ where $\mathrm{p}$ is prime. What does it become with our new definition? It remains true! Effectively : 


\section{Proof :}

We have $(2 m)^{2} \leq 4 m^{2}+1 \leq(2 m+1)^{2} \leq 4 m^{2}+8 m+1 \leq(2 m+2)^{2}$

But we will prove now that

$$
\begin{aligned}
& G C D\left(4 m^{2}+1,4 k^{2}+1\right)=5 ; m \neq k \\
& G C D\left(4 m^{2}+8 m+1,4 k^{2}+8 k+1\right)=3 ; m \neq k \\
& G C D\left(4 m^{2}+1,4 p^{2}+8 p+1\right)=1
\end{aligned}
$$

It is true for the two first asserstions and for the third, let us suppose $\mathrm{d}$ divising both the two equations, we have

$$
\begin{aligned}
& d\left|4 m^{2}+1 ; d\right| 4 p^{2}+8 p+1 \text { hence } \\
& d\left|4 p^{2}+8 p+1-4 m^{2}-1 \Rightarrow d\right| p^{2}-m^{2}+2 p \\
& d\left|4 p^{2}+8 p+1+4 m^{2}+1 \Rightarrow d\right| 2 p^{2}+2 m^{2}+4 p+1 \\
& =2(p+m)^{2}-2 p m+4 p+1 \\
& d \mid(p-m)(p+m)^{2}+2 p(p+m) \\
& d \mid 2(p-m)(p+m)^{2}-2 p m(p-m)+4 p(p-m) \\
& \Rightarrow d \mid-2 p m(p-m)+p-m=-2 p^{2} m+2 p m^{2}-p-m \\
& \Rightarrow d \mid-4 p^{2} m-8 p m-m+8 p m+m+4 p m^{2}+p \\
& \Rightarrow d|p m \Rightarrow d| p-m \Rightarrow d \mid m \Rightarrow d=1 \\
& \text { because } d \text { is odd. And } \\
& m=5\left(k+k^{\prime}\right) \pm 1 \\
& p=5\left(k-k^{\prime}\right) \pm 1 \neq m \\
& \Rightarrow 4 m^{2}+1=5(20(k+2 \pm 8(k-2) \\
& 4 p^{2}+1=5(20(k) \\
& \text { And } \\
& m=3(k+k) \\
& p=3(k-1-2)+2 \\
& 4 m^{2} \quad 8 m+1=(2 m-2)^{2}-3=\left(6\left(k+k^{\prime}\right)+6\right)^{2}-3=3 w \\
& 4 p^{2}+8,1=(2 p+2)^{2}-3=\left(6\left(k-k^{\prime}\right)+6\right)^{2}-3=3 w^{\prime}
\end{aligned}
$$

And $4 \mathrm{~m}^{2}+1$ and $4 \mathrm{~m}^{2}+8 \mathrm{~m}+1$ can be taken primes simultaneously with our definition of the primes, for example, the first $4 \mathrm{~m}^{2}+1$ divisible by 5 is for $\mathrm{m}=4$, and then $4 \mathrm{~m}^{2+} 1=65=13.5 \Rightarrow 5=65.13^{-1}$ is no more prime and 65 is prime, the second is for $m=6$ and then $4 \mathrm{~m} 2+1=145=29.5 \Rightarrow 29=145.5-1=$ 145.13.65-1is no more prime and 145 is prime, etc... until infinity. By the same way, the first $4 \mathrm{~m}^{2}$ $+8 \mathrm{~m}+1$ divisible by 3 is for $\mathrm{m}=2$ and then $4 m^{2}+8 m+1=33=11.3 \Rightarrow 3=33.11^{-1}$ is no more prime and 33 is prime, etc... until infinity; but $(2 m)^{2} \leq 4 m^{2}+1 \leq(2 m+1)^{2} \leq 4 m^{2}+8 m+1 \leq(2 m+2)^{2}$ 


\section{The Goldbach conjecture}

With the new definition $4 m^{2}+1,4 m^{2}+3$ and $4 p^{2}+8 p+1$ are always primes. Effectively

$$
\begin{aligned}
& d\left|4 m^{2}+1 ; d\right| 4 p^{2}+3 \Rightarrow d\left|2\left(p^{2}-m^{2}\right)+1 ; d\right| p^{2}+m^{2}+1 \\
& d \mid(p+m)^{2}-2 p m+1 \\
& d \mid(p-m)(p+m)^{2}-2 p m(p-m)+p-m \\
& d \mid 2(p-m)(p+m)^{2}+p+m \\
& \Rightarrow d \mid-4 p m(p-m)+2(p-m)-2 p-2 m \\
& =-4 m p^{2}-3 m+3 m+4 p m^{2}+p-p-4 m \\
& \Rightarrow d \mid m \Rightarrow d=1
\end{aligned}
$$

But

$\forall m|2 m=2(2 u+1) ; \exists m, p| u=m^{2}+p^{2}+2 p+1$

$\Rightarrow 2 m=4 m^{2}+1+4 p^{2}+8 p+1$

$\forall m|2 m=4 u ; \exists m, p| u=m^{2}+p^{2}+1$

$\Rightarrow 2 m=4 m^{2}+1+4 p^{2}+3$

So each even number is the sum of two primes.

\section{Back to the traditional definition of primes}

Let Goldbach true for the new definit d false or the old one, so

$\exists m|\forall p, q, \exists b| 2 m=p+q+2 b=b^{\prime}+q \quad b \neq 0, \mathrm{p}$ A are traditional primes, $\mathrm{p}$, q' new ones so $a \neq 0$

$$
\begin{aligned}
& 2 a x=q_{1}{ }^{\prime}+q_{2}{ }^{\prime} \\
& 2 a b=a\left(p^{\prime}+q^{\prime}-p-q=q_{1}\right. \\
& a=q_{1}{ }^{\prime}+q_{2}{ }^{\prime} \Rightarrow 2 b-1=-p-q
\end{aligned}
$$

it means that in side ere is an odd number, in the other an even one ! It is impossible $: b=0$. So the Goldbach con, re is try

Let no the 1 gendre o secture, we have found that for the new definition $\left.(2 n)^{2}+1\right)^{2}<q^{\prime}<(2 n+2)^{2}$

Where p', q wew primes. If Legendre conjecture is false for old and true for new primes 


$$
\begin{aligned}
& p-u x^{2}-(1-u)(1+x)^{2}=0 ; 0<u<1 \\
& p^{\prime}-u x^{2}-(1-u)(1+x)^{2}=b ; \forall p^{\prime} ; 0<u<1 \\
& a \neq 0 \\
& a^{2} p-u(a x)^{2}-(1-u)(a(1+x))^{2}=0=q-u(a x)^{2}-(1-u)(a(1+x))^{2} \\
& a^{2} p^{\prime}-u(a x)^{2}-(1-u)(a(1+x))^{2}=a^{2} b=a^{2}\left(p^{\prime}-p\right) \\
& =a^{2} p^{\prime}-q+\left(u^{\prime}-u\right) a^{2}\left(x^{2}-(x+1)^{2}\right) \\
& \Rightarrow-\frac{q}{a^{2}}=b-p^{\prime}+\left(u-u^{\prime}\right)\left(x^{2}-(x+1)^{2}\right) \in Z ; \forall a \\
& a=q \Rightarrow q=1
\end{aligned}
$$

\section{Conclusion}

We have generalized the definition of the primes and prov th egendre and the Goldbach conjectures for the generalization of the definition of primes rasonin allowed to prove that those conjectures are true for the o a definition too.

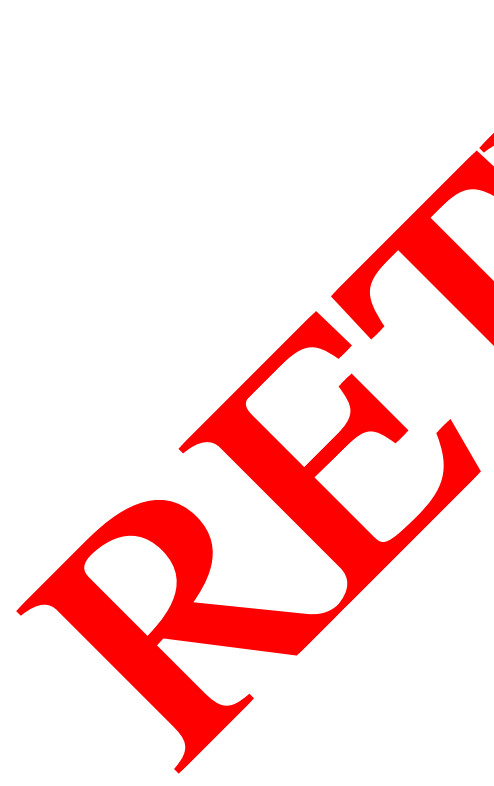

